\title{
Management of Chronic Achilles Tendon Injuries- Review of Current Protocols and Surgical Options
}

\author{
Madhu Periasamy ${ }^{1}$ Hari Venkatramani ${ }^{1}$ \\ ${ }^{1}$ Department of Plastic Surgery, Ganga Medical Centre and \\ Hospitals, Coimbatore, Tamil Nadu, India
}

\author{
Raja Sabapathy Shanmuganathan ${ }^{1}$
}

\begin{abstract}
Address for correspondence Shanmuganathan Raja Sabapathy, MS, MCh, DNB, Department of Plastic Surgery, Ganga Medical Centre and Hospitals, 313, Mettupalayam Road, Coimbatore, Tamil Nadu, India (e-mail: rajahand@gmail.com).
\end{abstract}

\begin{abstract}
Keywords

- reconstruction

- Achilles

- chronic

- tendon transfer

Chronic Achilles tendon injury is a challenging problem. A review of the different options available and their technical considerations is required. Reconstruction of the Achilles tendon depends on numerous factors such as the gap between the debrided tendon ends, the presence of a distal stump, and the and presence of scar tissue. Various protocols have been described for reconstruction. Methods of reconstruction vary from direct repair, bridging of the tendon gap with tendon grafts or tendon transfers. We review and enumerate the different protocols enumerated for the reconstruction of chronic Achilles tendon lesions in the literature and look at the authors preferred methods of reconstruction
\end{abstract}

\section{Introduction}

\section{Chronic Lesions of Achilles Tendon}

Lesions of the Achilles tendon are termed chronic if they are left untreated for more than 4 weeks. ${ }^{1}$ The cause of chronic Achilles tendon lesions includes neglected spontaneous ruptures and failed repairs of the Achilles tendon. A spontaneous rupture due to Achilles tendinopathy commonly occurs in middle-aged men who are occasionally active, and it often occurs without any warning signs or symptoms. ${ }^{2}$ The rupture most commonly occurs $\sim 2$ to $6 \mathrm{~cm}$ proximal to the insertion. ${ }^{3}$ The patient usually feels a sharp snap with severe pain on the posterior part of his ankle with swelling. It is immediately followed by an inability to walk or run and a sensation of imbalance and weakness on push off. The patient usually rests the affected part and once the signs of inflammation reduce, they slowly resume activity. In patient with diabetes, pain may not be a predominant symptom due to neuropathy and hence may cause the patient to ignore the acute rupture event and continue walking on the affected leg, causing further damage. The loss of the Achilles tendon results in a calcaneal limp 4 and this heel overload eventually causes a resistant heel ulcer in patients with an insensate foot. ${ }^{5}$

The Achilles tendon is a unique feature of man and is not found in any other member of the primate species. ${ }^{6}$ It most probably evolved as a result of the development of bipedal gait in the primitive Homo species. The evolution of the
Homo species has been attributed to its specialized ability for endurance running a unique feature absent in other nonhuman primate species. The ability to run requires long spring-like tendons which reduce the energy cost of transport by approximately $50 \%$. The most important of these springlike ligaments is the Achilles tendon.?

The Achilles tendon is formed by the fusion of the tendons of the two bellies of the gastrocnemius which lie superficially and that of the soleus muscle which lies deeper to the gastrocnemii. The respective tendons travel for some distance, separate from each other, and ultimately fuse to form a single tendon known as the Achilles tendon, the deeper fibers of which insert into the posterior part of the calcaneus and the superficial fibers continue onto become part of the plantar fascia. In the other great apes such as the gorilla, the gastrocnemius and soleus muscle tendons do not fuse but insert separately and directly into the calcaneum. ${ }^{6}$

Developmentally the lower limb bud rotates on itself during its formation. This is reflected in the anatomy of the Achilles tendon where the fibers of the gastrocnemius muscles are differentially rotated over the tendon of the soleus and are preferentially inserted onto the lateral side of the calcaneum, while the soleus fibers are inserted into the medial side of the calcaneum. This creates a wringing action of the Achilles tendon during contraction. ${ }^{8}$

The Achilles tendon inserts into the calcaneum at the Achilles tendon "enthesis organ." It is composed of the 
calcaneal periosseous fibrocartilage, the sesamoid fibrocartilage on the inner surface of the tendon, the retrocalcaneal bursa, and the Kager's fat pad. All these structures put together are called the Achilles tendon enthesis organ or synovio-entheseal complex ${ }^{9}$ and serve to dissipate the massive amount of torque transmitted through the Achilles tendon during activities such as jumping and running. A proportion of the Achilles tendon fibers passes on to the heel and becomes continuous with the plantar fascia without inserting into the calcaneum. This is representative of the myofascial web concept of Myers (Anatomy Trains) ${ }^{10}$ which is related to the concept that tendons seldom have an isolated insertion on to a single point of bony attachment, instead, they inset on to multiple adjacent structures to help spread out the load transmission.

Around the region of the enthesis, the Achilles tendon is surrounded by two bursae to reduce the friction during movement. A superficial bursa is found interposed between the skin and tendon, while the deep retrocalcaneal bursa is found between the tendon and the superior calcaneal tuberosity. ${ }^{11}$

The blood supply of the Achilles tendon can be divided into thirds. The primary vessel supplying the Achilles tendon is the posterior tibial artery which sends branches through a mesotenon like membrane which then expands and forms a paratenon with plexus of vessels. The upper third is also supplied from the gastrocnemius and soleus muscle bellies and the lower third from the periosteal plexus over the calcaneum and branches. Other studies have also identified that the Achilles tendon is supplied by two arteries, the middle third supplied by the peroneal artery, and the proximal and distal thirds being supplied by the posterior tibial artery. ${ }^{12}$

\section{Diagnosis}

A chronic Achilles tendon injury may present with an obvious sign of rupture with a palpable or visible defect in the continuity of the Achilles tendon (-Fig. 1). Clinical tests for testing the integrity of the Achilles tendon include the Simmond's or Thompson's calf squeeze test, Matle's test, O'Brien's needle test, and the Copeland's sphygmomanometer Test.

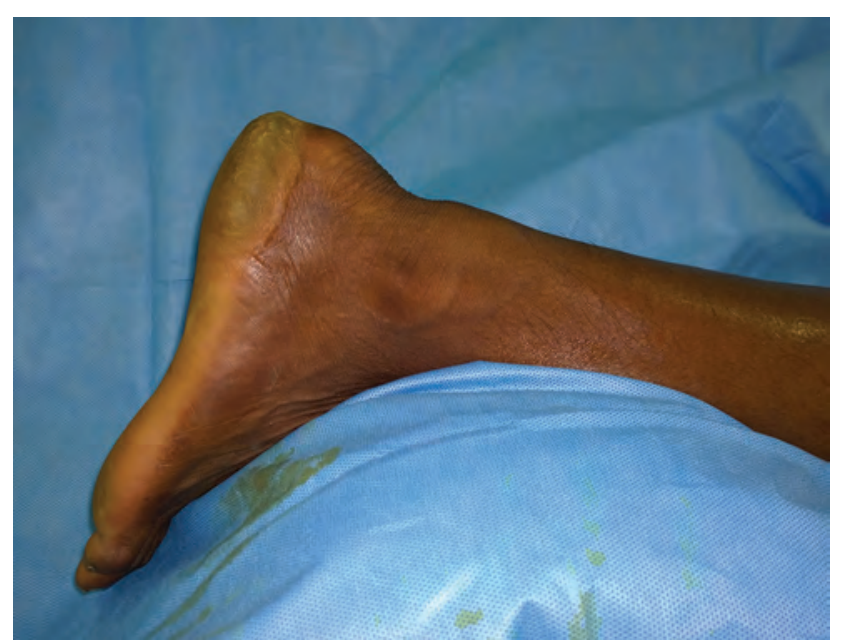

Fig. 1 Step like deformity visible on the lateral view. If a scar bridges the defect, such a dip may not be visualized.
The calf squeeze test described by Simmond ${ }^{13}$ about 5 years before Thompson ${ }^{14}$ involves squeezing of the calf muscles in a patient lying prone with his feet overhanging the edge of the examination couch. The examiner squeezes calf causing the feet to plantarflex indicating the integrity of the tendon. In the Matle's test, ${ }^{15}$ the patient lies prone with his knees flexed to 90 degrees and ankles let loose. In a patient with a ruptured Achilles tendon, the involved ankle falls into dorsiflexed or neutral position, while the normal ankle stays in plantarflexion due to the tone of the triceps surae (-Fig. 2).

In the O'Brien's needle test, ${ }^{16}$ a needle is inserted into the substance of the Achilles tendon $\sim 10 \mathrm{~cm}$ proximal to the insertion and just medial to the midline and the patient is asked to dorsiflex and plantarflex his ankle. The tip of the needle tilts distally and hub proximally with dorsiflexion if the substance of the tendon distal to the needle is intact. In the Copeland's sphygmomanometer test, ${ }^{17}$ a cuff is tied around the patient's midleg flexed at the knee in a prone position with the foot plantarflexed and is inflated to $100 \mathrm{~mm} \mathrm{Hg}$. The patient's foot is then passively dorsiflexed and the quantum of the rise in the sphygmomanometer is noted. In individuals with an intact Achilles tendon, the pressure will rise to $\sim 140 \mathrm{~mm} \mathrm{Hg} .{ }^{17} \mathrm{~A}$ point to remember, however, is that the test was originally described using a mercury manometer which is a rarity nowadays.

During clinical testing, it must be kept in mind that a false positive result may occur due to the formation of a bridging scar in between the cut ends of the Achilles tendon. Patients are also able to perform an active ankle flexion the presence of a ruptured Achilles tendon due to the action of the intact secondary flexors of the ankle joint such as the flexor hallucis longus (FHL), flexor digitorum longus, peroneus longus, and tibialis posterior muscles. This can be unmasked by the relative weakness of the affected side when compared with the normal side.

\section{Imaging}

A plain X-ray taken in an individual shows the soft tissue outline of the Achilles tendon inserting into the calcaneum (-Fig. 3). The maximum anteroposterior width of the

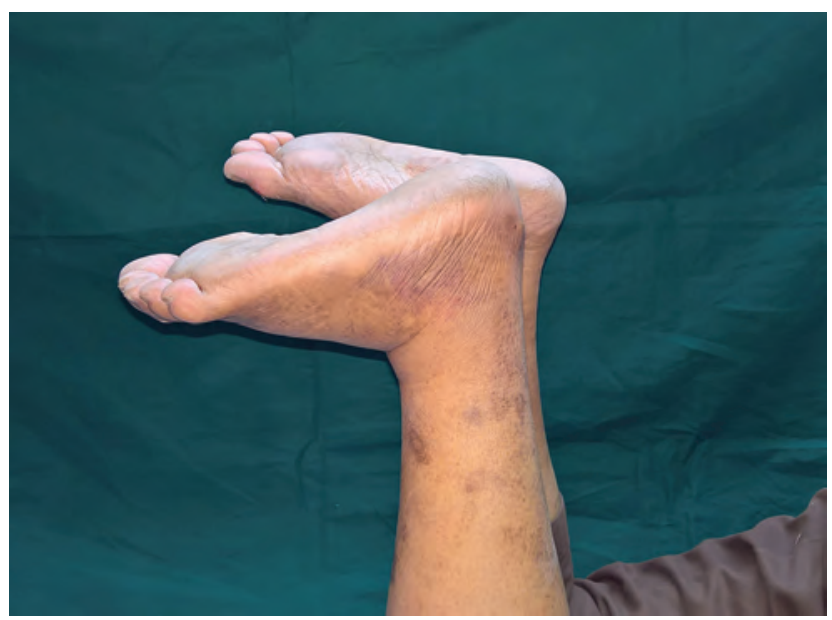

Fig. 2 Matle's test: The involved ankle in the foreground is more dorsiflexed compared with the uninvolved foot in the back ground which remains plantarflexed due to the tone of the triceps surae. 


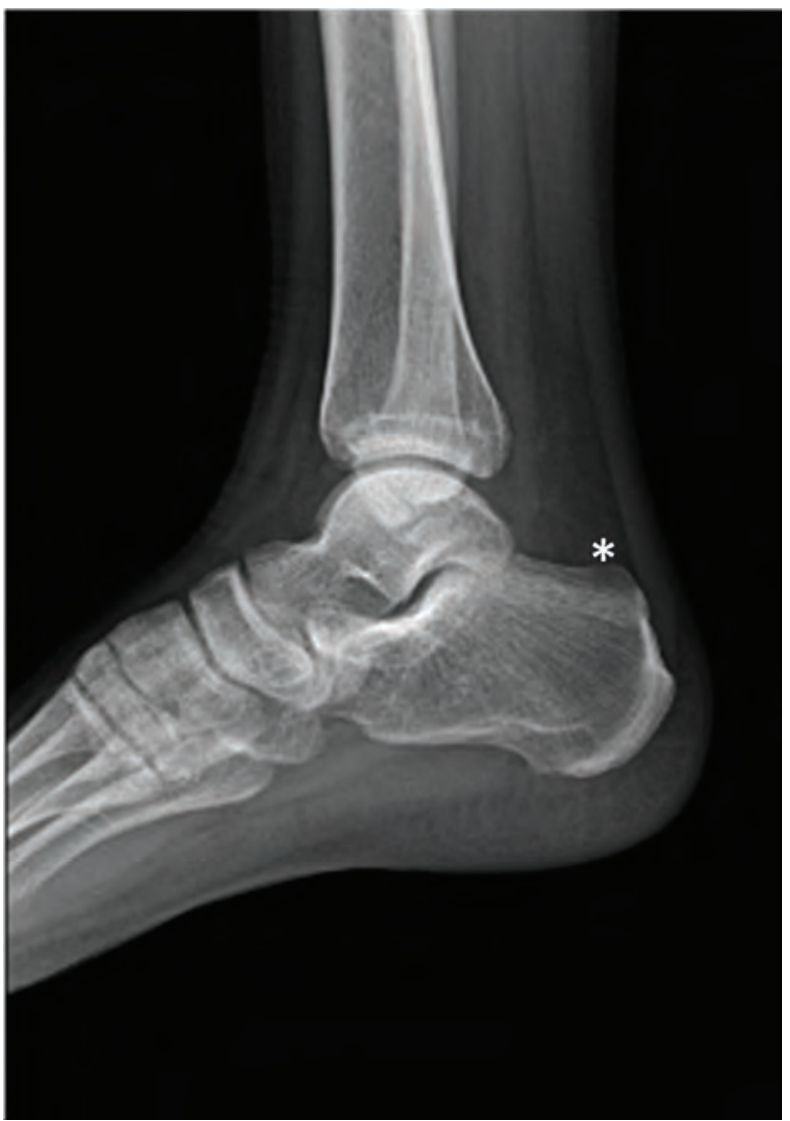

Fig. 3 The X-ray shows the soft tissue outline of the normal Achilles tendon with a lucent area deep to it representing the Kager fat pad $\left.{ }^{*}\right)$ and fatty tissue deep to the Achilles tendon.

Achilles tendon in the lateral view radiograph has been found to be $8 \mathrm{~mm}$ and anything more than that signifies a pathology of the Achilles tendon. ${ }^{18} \mathrm{~A}$ translucent area just beneath the Achilles tendon represents the fat pad and it dips $\sim 2 \mathrm{~mm}$ below the superior most border of the calcaneus. In inflammatory conditions of the Achilles tendon, this inverted triangle bordered by the calcaneum anteriorly, the enthesis posteriorly and continuous with the Kager's fat pad superiorly, gets obliterated.
High-frequency ultrasonogram can also be used to identify the continuity of the Achilles tendon, its dimensions, and its vascularity. ${ }^{19}$ The gold standard imaging test is a magnetic resonance imaging (MRI) scan of the involved area. T1-weighted images show a good anatomical delineation of the tendon, whereas T2-weighted images demonstrate fluid accumulation, a sign of tendon pathology, well. ${ }^{20}$ The normal average thickness of the Achilles tendon on an MR is $\sim 6 \mathrm{~mm} .{ }^{21}$ The normal retrocalcaneal bursa measures $\sim 6 \mathrm{~mm}$ superoinferiorly, $3 \mathrm{~mm}$ mediolaterally, and $2 \mathrm{~mm}$ anteroposteriorly. ${ }^{11}$

\section{Pedobarogram}

A pedobarogram is a dynamic recording of the pressures that act on the sole of the foot during the stance phase of the gait cycle. Information like peak pressures, force, area in contact with the ground, and the duration of the stance phase can be gathered from a pedobarogram. Force plate testing has not been described as a diagnostic modality of Achilles tendon injuries; however, it has been an established method to diagnose gait disturbances in patients with neuropathic ulcers and orthopaedic problems..$^{22-25}$

We have noticed in our series of patients that the pedobarogram is effective at indirectly diagnosing an inefficiency of the muscle-Achilles tendon-calcaneum, midfoot, and forefoot axis integrity by documenting the increase in calcaneal loading and the associated calcaneal gait that occurs with Achilles tendon insufficiency ${ }^{26}$ even when clinical testing is inconclusive because of fibrous bridging of the gap. Serial pedobarograms at regular intervals after surgical repair of a ruptured Achilles tendon documented the return of function evidenced by the progressive increase in forefoot loading ( - Fig. 4 ).

\section{Management Protocols}

Reconstruction of the chronic Achilles tendon injury primarily depends on the age of the injury and the magnitude of the gap that exists between the proximal tendon that has undergone adaptive shortening and the distal healthy tendon. The length of the final gap is

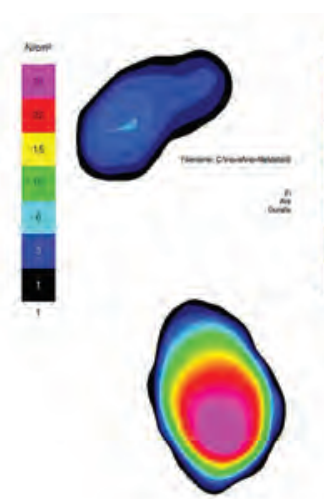

a
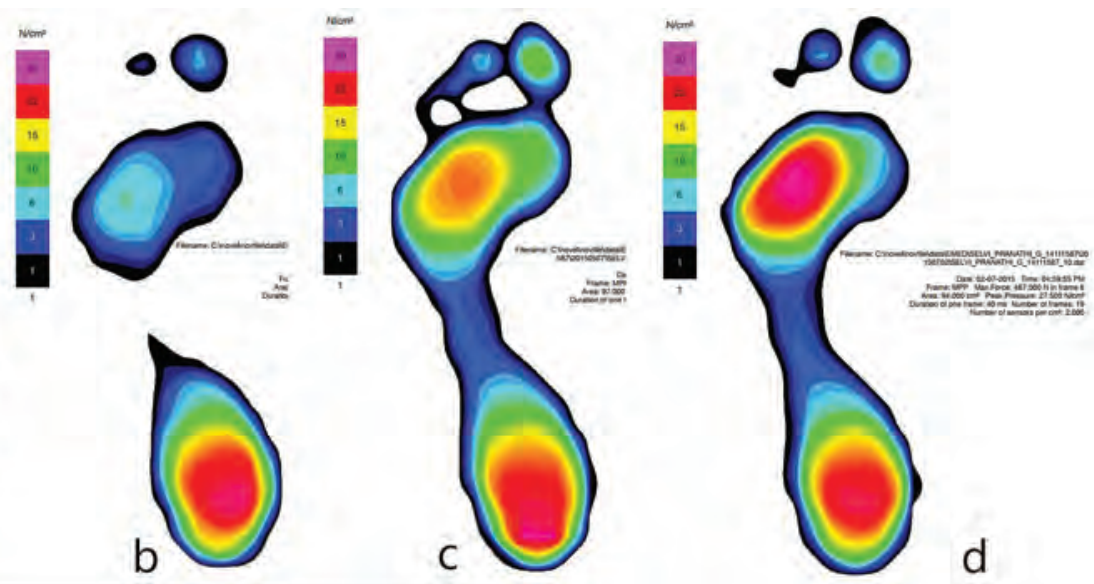

Fig. 4 Serial pedobarogram in a young girl after direct repair of an acute Achilles tendon injury. Image (A) showing only calcaneal loading with little or no forefoot loading. Images (B-D) showing the gradual return of forefoot function with a concomitant reduction in heel pressures. 
determined after debridement of the fibrotic and degenerate areas of the ruptured tendon ends. Most protocols for repair of chronic Achilles tendon injuries take into consideration the size of the defect as the lone criterion. More recent publications have stressed the need to take the age of the injury, the status of the tendon ends, and other factors into consideration.

The Myerson protocol ${ }^{27}$ divides chronic Achilles tendon injuries based on the size of the defect. Lesions with a gap of less than $2 \mathrm{~cm}$ are directly repaired. A gap of 2 to $5 \mathrm{~cm}$ are treated by a $\mathrm{V}$-Y advancement with an occasional tendon transfer for augmentation if required.

Gaps of greater than $5 \mathrm{~cm}$ are primarily treated with a tendon transfer with a combination of a V -Y lengthening if required.

The Kuwada protocol ${ }^{28}$ divides the lesions into four types. Type I consists of partial thickness injuries which were treated conservatively. Type II consists of defects up to $3 \mathrm{~cm}$ and are treated with an end-to-end repair. Type III lesions, those with gaps of 3 to $6 \mathrm{~cm}$ between the ends of debrided tendon, require a tendon graft flap possibly strengthened with a synthetic graft. Type IV lesions with greater than $6 \mathrm{~cm}$ were treated with a gastrocnemius recession and free tendon graft or synthetic graft.

Maffulli et al ${ }^{29}$ gave no specific protocol based on the size of the defect. They suggested that the primary end-to-end repair whenever possible was the best choice and a tendon transfer was to be done for defects that could not be repaired primarily. The first choice for a transfer was a peroneus brevis followed by the flexor digitorum longus muscle with tendon for larger defects. In cases where the tear was larger than $6.5 \mathrm{~cm}$, a nonvascularized gracilis tendon graft or semitendinosus graft was preferred.

Other protocols that have been published for the treatment of chronic Achilles tendon lesions based on the size of the include those by Bevialcqua, ${ }^{30}$ Den Hartog, ${ }^{31}$ and Krahe and Berlet. ${ }^{32}$

Recently, Buda et $\mathrm{al}^{33}$ have published a protocol which considers other factors like the extent of injury, time since injury, the presence of MRI signs of tendinopathy like paratenonitis, tendinosis of the ruptured ends, and presence of insertional tendinopathy apart from the size of the defect. They suggested an end-to-end repair of full-thickness defects of 1 to $2 \mathrm{~cm}$ in size. For defects $>2 \mathrm{~cm}$ up to $5 \mathrm{~cm}$, they suggested a turndown flap or $\mathrm{V}-\mathrm{Y}$ advancement repair. One or two tendon transfers with a $\mathrm{V}-\mathrm{Y}$ tendinous flap and the use of an allograft were recommended in gaps larger than $5 \mathrm{~cm}$.

\section{Operative Management and the Authors' Preferred Techniques}

\section{Primary Repair}

Primary repair with or without a gastrocsoleus lengthening is the preferred procedure when the gap between healthy tendon ends after debridement is less than $2 \mathrm{~cm}$ and the distal insertion of the Achilles tendon on to the calcaneum is intact. The operation is usually done under spinal anesthesia and the patient is positioned in a semiprone position turned to the involved side which brings the operative site upward and gives unrestricted access to the posterior, medial, and lateral sides. The leg is placed on a foam pad with mild flexion at the knee to allow relaxation of the gastrocnemii muscles. The Achilles tendon is approached through a curved incision raising a medially based flap and extended proximally and distally in the midline giving good access to the proximal and distal stumps and enthesis. The most commonly studied suture techniques include the double-stranded Kessler, double-stranded Bunnell, and Krackow repairs. The Krackow technique has shown a greater tendency to gap formation. ${ }^{34}$ The actual strength of the repairs has been shown to depend on the number of suture strands crossing the repair site rather than the suture technique as such. ${ }^{35}$ We prefer the double-stranded Kessler repair with a thick monofilament 1 Prolene (NW 843- Ethicon), though newer materials like Ethibond (Ethicon) and Fiberwire (Arthrex) have been shown to have greater tensile strength in recent studies..$^{34}$

When the enthesis is grossly destroyed by degenerative changes and the gap is lesser than $2 \mathrm{~cm}$, the proximal tendon end is fixed on to the decorticated surface of the posterior part of the calcaneus with the help of a suture anchor. This repair may or may not be strengthened with a tendon transfer depending upon the tension in the repair.

Occasionally, a V-Y lengthening or gastrocsoleus lengthening may be required to bring down the proximal tendon to enable a primary repair. We prefer a transverse Vulpius gastrocsoleus recession ${ }^{36}$ done just below the insertion of the gastrocnemii. A transverse incision is made along the gastrocsoleus leaving the soleus muscles intact to fractionally lengthen the tendon by $\sim 1$ to $2 \mathrm{~cm}$. The division of the midline raphe of the soleus helps to gain a few millimeters. The same release made in the form of a $V$ can be reapproximated in the form of a $\mathrm{Y}$ to get a $\mathrm{V}-\mathrm{Y}$ lengthening.

\section{Peroneus Brevis Transfer}

Some patients with an intact distal stump may require an augmentation of the primary repair with a tendon transfer. The peroneus brevis tendon transfer is the transfer of choice for augmentation of a primary repair. The tendon is harvested through an incision at the base of the fifth metatarsal and taken out through the surgical wound behind the fibula. The peroneus longus tendon lying superficial to the brevis tendon is left intact. The tendon length is relatively short but is enough to be inserted into the calcaneum in a dorsoplantar direction using an interference screw and can also be also hitched to the distal stump of the tendoachilles. ${ }^{37}$ Considering the shorter tendon length and need for an interference screw, we prefer to use the peroneus brevis as a distally based flap for cover of an incomplete Achilles tendon injury rather than a proximally based donor for transfer.

\section{Flexor Hallucis Longus Transfer}

This is our preferred technique for tendon transfers for both substitution and augmentation of Achilles tendon repairs. FHL tendon transfer for Achilles tendon reconstruction was first described by Wapner et al. ${ }^{38}$ In patients with an enthesis loss and proximal tendon of insufficient length to 


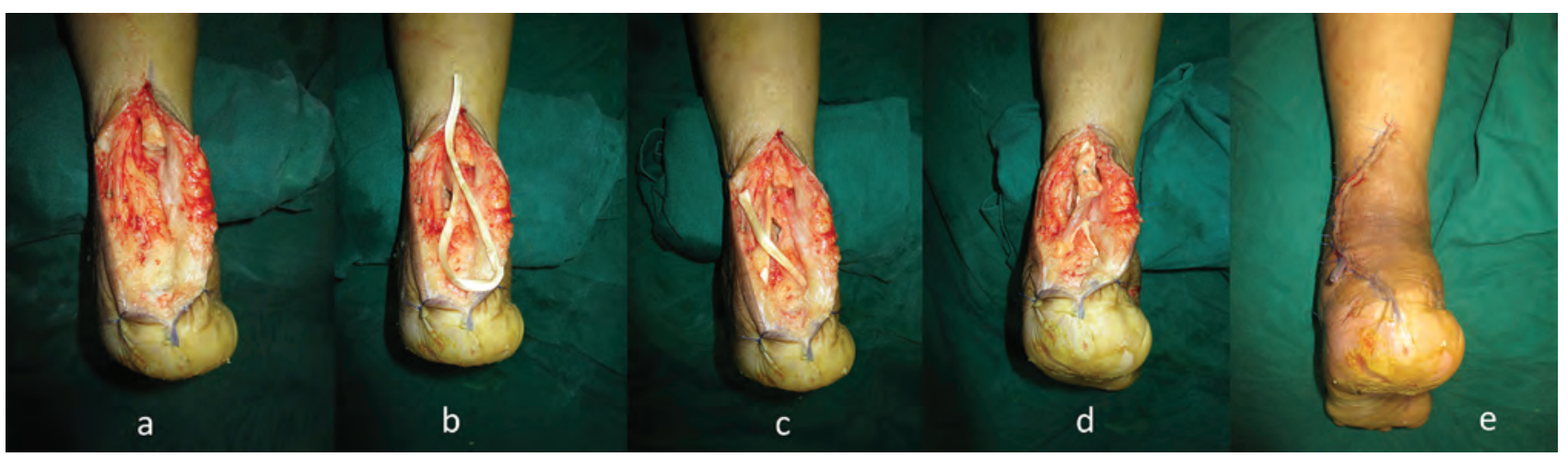

Fig. 5 Transfer of the flexor hallucis longus tendon to bridge a gap of the Tendo Achilles. The tendon is harvested from the base of the toe using a two-incision technique (A) and routed through the calcaneus and woven into the proximal Tendo Achilles stump (B-D). Skin is closed over a drain (E).

reach the calcaneum, the FHL transfer is a preferred procedure to both create a new insertion and bridge the gap in the Achilles tendon ( - Fig. 5). The FHL tendon harvested at the base of the great toe (double incision) is an ideal choice for transfer in this situation. Some authors prefer to use the single incision technique to harvest the FHL proximal to the Henry knot to preserve great toe function at the cost of donor tendon length. The muscle is synergistic and the strongest of all available transfers. ${ }^{39}$ The FHL muscle and tendon have been proven to hypertrophy following transfer and achieve a concentric and eccentric contraction strength comparable to that of the uninjured side. ${ }^{40}$

The FHL is first isolated at the base of the great toe and at the midfoot through separate incisions. The distal end is cut and retrieved at the midfoot. The proximal part of the muscle belly with its tendinous origin lies in the midline just anterior to the ruptured ends of the Achilles tendon. The deep fascia separating the deep compartment from the Achilles tendon is incised longitudinally to identify the FHL and which is then isolated. The tendon is again retrieved behind the ankle after dividing the Henry knot. The tendon is then routed through a transosseous tunnel in the calcaneum and sutured onto itself in a Pulvertaft weave with the ankle in $~ 45$ degrees of flexion. The distal free end is then woven mediolaterally into the proximal end of the Achilles tendon. Each weave is anchored with 3-0 Prolene (Ethicon) sutures. Once the repair is complete, the skin flap is sutured over a tube drain. The foot is dressed, and the ankle position is maintained with an anterior below knee slab for $\sim 2$ weeks.

\section{Postoperative Protocols}

After suture removal, the repair was protected in a below knee equinus cast for $\sim 2$ weeks and a neutral cast for $\sim 8$ weeks. The patients are allowed protected full weight bearing with a below knee cast on the operated leg 8 weeks after surgery and unprotected full weight bearing is allowed at 12 weeks post-surgery in footwear with a heel raise. The patients are cautioned not to attempt isolated forefoot weight bearing particularly when climbing stairs. Ankle plantar flexion strengthening exercises are started and the patient reviewed once in 2 months. The serial pedobarographic evaluation showed the return of forefoot loading in $\sim 11$ to 13 months in patients who underwent an FHL transfer. Other tendons transfers reported in the literature include the flexor digitorum longus muscle transfer. On comparison of the FHL and the peroneus brevis muscles in cadaveric studies, Sebastian et al concluded that the mechanical properties both muscles were similar. ${ }^{41}$

\section{Free Tendon Graft}

Few patients have very large gaps due to loss of tendon substance which is not amenable for repair by tendon flaps. They may be reconstructed with free tendon grafts. Commonly used free tendon grafts include the semitendinosus tendon, gracilis tendon, ${ }^{42}$ FHL tendon graft, and fascia lata. We prefer to use the fascia lata (-Fig. 6). It can be rolled into a thick cord like graft and then woven into the proximal and distal ends of the Achilles tendon using a Pulvertaft weave. The use of allograft tendon ${ }^{43}$ alone or in combination with a xenograft ${ }^{44}$ has also been described in the repair of large gaps, but this procedure is not commonly described in the literature and its use is an exception.

\section{Achilles Tendon Gap with Overlying Soft Tissue Loss}

Sometimes a chronic lesion occurs because of a failed primary repair of an acute Achilles tendon repair. Such lesions usually present with loss of the overlying skin and soft tissue. In such cases, we prefer to do a debridement of the sloughed tissues till healthy bleeding edges. The bed and enthesis are debrided to healthy tissue and all calcific degenerate tendon tissues are removed. The wound is managed by moist dressing till a healthy clean bed is achieved. In the next stage, a tendon reconstruction is done with a tendon graft or FHL tendon transfer with wound closure by a microvascular gracilis muscle flap cover which is the flap of choice in our unit ( $\mathbf{- F i g . ~ 6 ) . ~ T h e ~}$ muscle is covered with a split-thickness skin graft. Other flaps that have been described for use in chronic Achilles tendon lesions with soft tissue loss include a local reverse sural pedicled flap, perforator based propeller flaps ${ }^{45}$ ( - Fig. 7), reverse peroneus brevis flaps, ${ }^{46}$ and the microvascular free anterolateral thigh flap and latissimus dorsi flap. ${ }^{47-49}$ In a rare case with infected complex defect, reconstruction with a free gracilis flap alone has been found to bridge the gap and function as an effective interposition graft. ${ }^{50}$ 


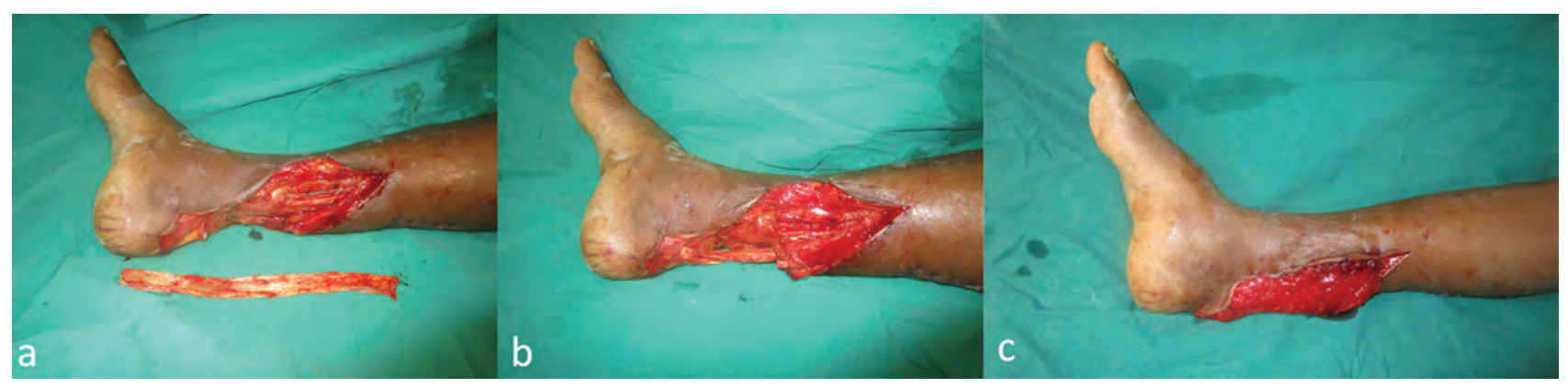

Fig. 6 In very large gaps, a fascia lata graft (A) is used to bridge the gap (B). In cases with concomitant soft tissue loss, a free gracilis muscle flap is used to provide cover (C).

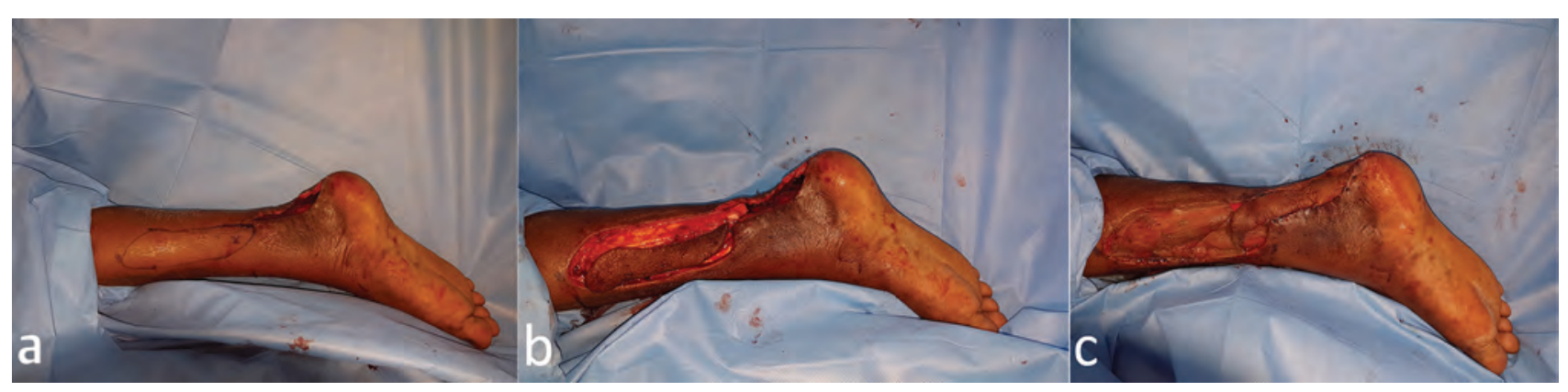

Fig. 7 Propeller flap based on the posterior tibial perforator used to cover a post debridement defect following a failed reconstruction of a chronic Tendo Achilles injury.

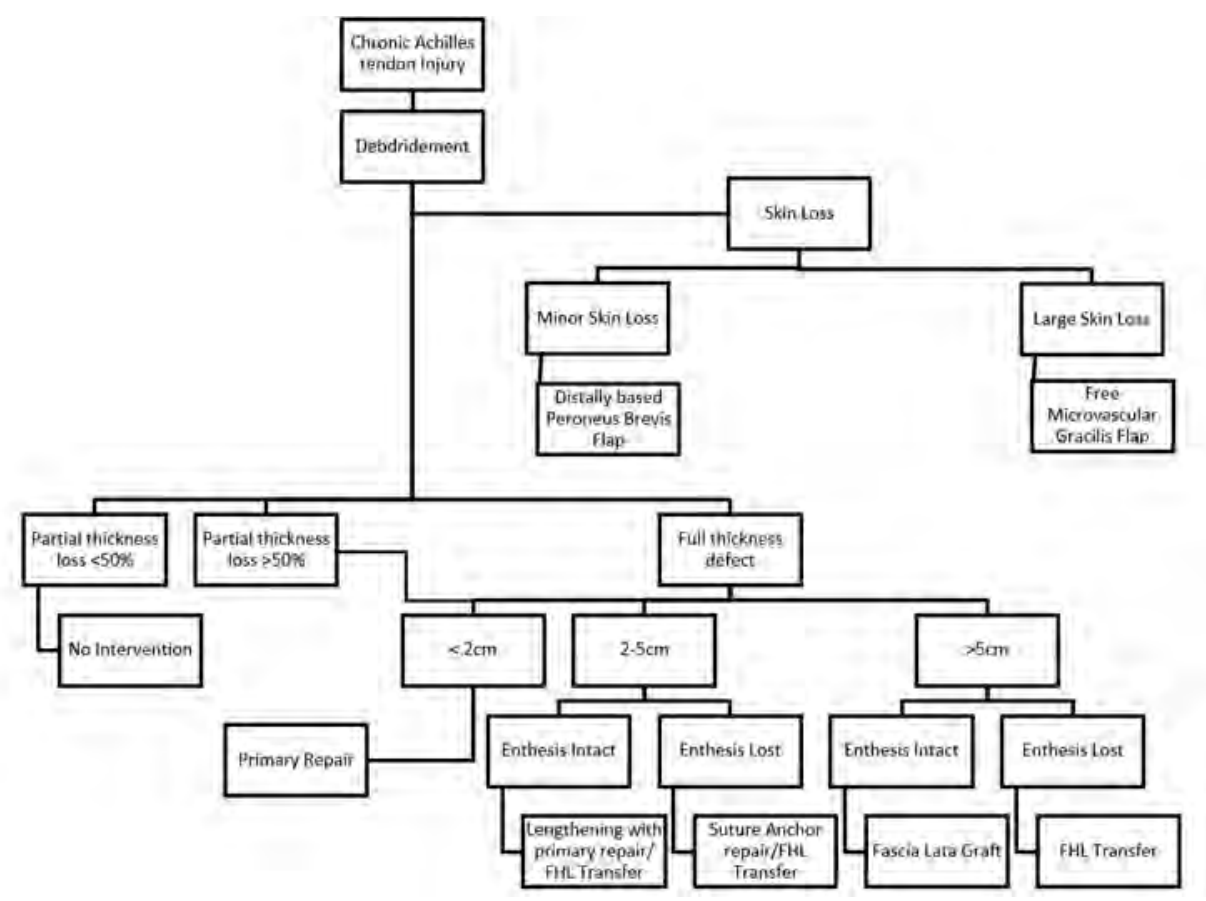

Fig. 8 Flowchart of the authors protocol of management of chronic Achilles tendon Injuries. FHL, flexor hallucis longus.

\section{Overview}

Repair and reconstruction of this biomechanically important tendon depend upon multiple factors which need to be taken into consideration. Following one of the multiple protocols
( - Fig. 8) which best suits the reconstructing surgeon and the clinical scenario with adherence to principles of tendon repair and reconstruction, namely a good debridement, gentle appropriate technique and ensuring that the repair is covered by healthy soft tissue should ensure good results. 


\section{Conflict of Interest}

None declared.

\section{References}

1 Flint JH, Wade AM, Giuliani J, Rue JP. Defining the terms acute and chronic in orthopaedic sports injuries: a systematic review. Am J Sports Med 2014;42(1):235-241

2 Hattrup SJ, Johnson KA. A review of ruptures of the Achilles tendon. Foot Ankle 1985;6(1):34-38

3 Jonathan S. Young, Maffulli N. Chronic ruptures of the Achilles tendon. In: Nicola Maffulli, Louis C. Almekinders, eds. The Achilles Tendon. London: Springer-Verlag London Limited; 2007:165-171

4 Gabel S, Mandi A. Neglected rupture of the Achilles tendon. Foot Ankle Int 1994;15(9):512 -517

5 Chilvers M, Malicky ES, Anderson JG, Bohay DR, Manoli A. Heel overload associated with heel cord insufficiency. Foot Ankle Int 2007;28(6):687-689

6 Klenerman L. The history of the tendo Achillis and its rupture. In: Maffuli N, Almekinders LC, eds. The Achilles Tendon. London: Springer; 2007:1

7 Bramble DM, Lieberman DE. Endurance running and the evolution of Homo. Nature 2004;432(7015):345-352

8 Benjamin M, Theobald P, Suzuki D, Toumi H. The anatomy of the Achilles tendon. In: Nicola Maffulli LCA, ed. The Achilles tendon. London: Springer-Verlag London Limited; 2007:5-16

9 Benjamin M, McGonagle D. The enthesis organ concept and its relevance to the spondyloarthropathies. In: Lopez-Larrea C, Diaz-Pena R, eds. New York, NY: Springer; 2009:57-70

10 Myers TW. Anatomy Trains. New York: Churchill Livingstone; 2001:137-164

11 Frey C, Rosenberg Z, Shereff MJ, Kim H. The retrocalcaneal bursa: anatomy and bursography. Foot Ankle 1992;13(4):203-207

12 Chen TM, Rozen WM, Pan WR, Ashton MW, Richardson MD, Taylor GI. The arterial anatomy of the Achilles tendon: anatomical study and clinical implications. Clin Anat 2009;22(3):377-385

13 Simmonds FA. The diagnosis of the ruptured Achilles tendon. Practitioner 1957;179(1069):56-58

14 Thompson TC. A test for rupture of the tendo Achilles. Acta Orthop Scand 1962;32:461-465

15 Matles AL. Rupture of the tendo Achilles: another diagnostic sign. Bull Hosp Jt Dis 1975;36(1):48-51

16 O'Brien T. The needle test for complete rupture of the Achilles tendon. J Bone Joint Surg Am 1984;66(7):1099-1101

17 Copeland SA. Rupture of the Achilles tendon: a new clinical test. Ann R Coll Surg Engl 1990;72(4):270-271

18 Bleakney RR, White LM, Maffulli N. Imaging of the Achilles tendon. In: The Achilles Tendon. London: Springer-Verlag London Limited; 2007:25-36

19 Roesch HJ, Milanese S, Osborne B, Spurrier DJ, Thoirs KA. The acute effects of exercise on tendon dimensions and vascularity. An exploratory study using diagnostic ultrasound of the male Achilles tendon. J Sci Med Sport 2018;21(10):982-987

20 Rosenberg ZS, Beltran J, BencardinoJT. From the RSNA Refresher Courses. Radiological Society of North America. MR imaging of the ankle and foot. Radiographics 2000;20(Spec No): S153-S179

21 Marshall H, Howarth C, Larkman DJ, Herlihy AH, Oatridge A, Bydder GM. Contrast-enhanced magic-angle MR imaging of the Achilles tendon. Am J Roentgenol 2002;179:187-192

22 Skopljak A, Muftic M, Sukalo A, Masic I, Zunic L. Pedobarography in diagnosis and clinical application. Acta Inform Med 2014;22(6):374-378
23 Ozgul B, Timurtas E, Demirbuken I, Polat MG. Dynamic pedobarography in evaluation of prone and neutral foot in adolescent. Gait Posture 2016;49:179

24 Hughes J. The clinical use of pedobarography. Acta Orthop Belg 1993;59(1):10-16

25 Hutchinson RJ, Betts RP, Donnan LT, Saleh M. Assessment of Ilizarov correction of club-foot deformity using pedobarography. A preliminary report. J Bone Joint Surg $\mathrm{Br}$ 2001;83(7):1041-1045

26 Nishimoto GS, Attinger CE, Cooper PS. Lengthening the Achilles tendon for the treatment of diabetic plantar forefoot ulceration. Surg Clin North Am 2003;83(3):707-726

27 Myerson MS. Achilles tendon ruptures. Instr Course Lect 1999;48:219-230

28 Kuwada GT. Diagnosis and treatment of Achilles tendon rupture. Clin Podiatr Med Surg 1995;12(4):633-652

29 Maffulli N, Ajis A, Longo UG, Denaro V. Chronic rupture of tendo Achillis. Foot Ankle Clin 2007;12:583-596

30 Bevilacqua NJ. Treatment of the neglected Achilles tendon rupture. Clin Podiatr Med Surg 2012;29(2):291-299, viii

31 Den Hartog BD. Surgical strategies: delayed diagnosis or neglected Achilles' tendon ruptures. Foot Ankle Int 2008;29(4):456-463

32 Krahe MA, Berlet GC. Achilles tendon ruptures, re rupture with revision surgery, tendinosis, and insertional disease. Foot Ankle Clin 2009;14(2):247-275

33 Buda R, Castagnini F, Pagliazzi G, Giannini S. Treatment algorithm for chronic Achilles tendon lesions: review of the literature and proposal of a new classification. J Am Podiatr Med Assoc 2017;107(2):144-149

34 Kim J, Rusly J, Fruscello K, et al. Comparison of Achilles Tendon Suture Repair Techniques: Krackow vs Modified Mason-Allen Under Cyclic Loading in an In-Vitro Bovine Model. ORS 2012 Annu Meet 2012;(1896):2012

35 McCoy BW, Haddad SL. The strength of Achilles tendon repair: a comparison of three suture techniques in human cadaver tendons. Foot Ankle Int 2010;31(8):701-705

36 Vulpius O, Stoffel A. Tenotomie der end schen der $\mathrm{mm}$ gastrocnemius el soleus mittels rutschenlassens nach vulpius. In: Orthopadische Operationslehre. Stuttgart (Germany): Nabu Press; 1913:29-31

37 Maffulli N, Oliva F, Costa V, Del Buono A. The management of chronic rupture of the Achilles tendon: minimally invasive peroneus brevis tendon transfer. Bone Joint J 2015;97-B(3):353-357

38 Wapner KL, Pavlock GS, Hecht PJ, Naselli F, Walther R. Repair of chronic Achilles tendon rupture with flexor hallucis longus tendon transfer. Foot Ankle 1993;14(8):443-449

39 Kim J-Y, Lee I, Seo K, Jung W, Kim B. FHL tendon transfer in diabetics for treatment of non-healing plantar heel ulcers. Foot Ankle Int 2010;31(6):480-485

40 Ozer H, Ergisi Y, Harput G, Senol MS, Baltaci G. Shortterm results of flexor hallucis longus transfer in delayed and neglected Achilles tendon repair. J Foot Ankle Surg 2018;57(5):1042-1047

41 Sebastian H, Datta B, Maffulli N, Neil M, Walsh WR. Mechanical properties of reconstructed Achilles tendon with transfer of peroneus brevis or flexor hallucis longus tendon. J Foot Ankle Surg 2007;46(6):424-428

42 Maffulli N, Oliva F, Maffulli GD, Buono AD, Gougoulias N. Surgical management of chronic Achilles tendon ruptures using less invasive techniques. Foot Ankle Surg 2018; 24(2):164-170

43 Cienfuegos A, Holgado MI, Díaz del Río JM, González Herranz J, Lara Bullón J. Chronic Achilles rupture reconstructed with Achilles tendon allograft: a case report. J Foot Ankle Surg 2013;52(1):95-98 
44 Hollawell S, Baione W. Chronic Achilles tendon rupture reconstructed with Achilles tendon allograft and xenograft combination. J Foot Ankle Surg 2015;54(6):1146-1150

45 Vaienti L, Calori GM, Leone F, Brioschi M, Parodi PC, Marchesi A. Posterior tibial artery perforator flaps for coverage of Achilles region defects. Injury 2014;45(Suppl 6):S133-S137

46 Lorenzetti F, Lazzeri D, Bonini L, et al. Distally based peroneus brevis muscle flap in reconstructive surgery of the lower leg: postoperative ankle function and stability evaluation. J Plast Reconstr Aesthet Surg 2010;63(9):1523-1533

47 Upadhyaya DN, Khanna V, Kohli R, Tulsi SP, Garg S. Functional reconstruction of complex tendo Achilles defect by free latissimus dorsi muscle flap. Indian J Plast Surg 2012;45(3):572-575
48 Jepegnanam TS, Nithyananth M, Boopalan PRJVC, Cherian VM, Titus VT. Reconstruction of open contaminated Achilles tendon injuries with soft tissue loss. J Trauma 2009;66(3):774-779

49 Lee JW, Yu JC, Shieh SJ, Liu C, Pai JJ. Reconstruction of the Achilles tendon and overlying soft tissue using antero-lateral thigh free flap. Br J Plast Surg 2000;53(7):574-577

50 Sabapathy SR, Venkataramani H, Latheef L, Bhardwaj P. Reconstruction of segmental defects of Achilles tendon: is it a must in infected complex defects? Indian J Plast Surg 2013;46(1):121-123 